\title{
Preface
}

\section{Commingled Remains Special Issue}

\author{
John E. Byrd ${ }^{a^{*}}$
}

This special issue of Forensic Anthropology is another contribution to a growing literature aimed at solving what may be the most vexing problem in human identification: largescale commingling of human remains. The contributors to this issue provide us with new ideas concerning methodological approaches, as well as insights emerging from the successful resolution of some of the world's most difficult identification problems. While the applications center on anthropology, the lessons are more broadly applicable to current-day disasters in which large numbers of fragmented remains must be identified. The central theme running throughout this special issue is that no single discipline is the answer to the problem. Complex cases require sophisticated solutions.

The resolution of any case involving commingled human remains (CHR) requires the employment of a variety of skills, often from more than one forensic discipline. It is now common to select and submit DNA samples from remains early in the process and to defer further work until the DNA laboratory results are in hand. Here we see a handoff from the anthropologist (or pathologist as the case may be) to the DNA scientists, and then back to the anthropologist. We need the expert understanding of anatomy and experience sorting remains to select the samples, along with DNA expertise to interpret the DNA findings. Large CHR cases $(N>4)$ create problems of scale that are not of general concern with small cases. Methods with modest error rates or random match probabilities (e.g., mtDNA) lose power rapidly as the number of individuals increases. Therefore, large CHR cases force the application of more methods simultaneously and motivate the development of new methods for sorting commingled remains.

The fundamental challenge of estimating the number of commingled persons is addressed in this issue with the

${ }^{a}$ Defense POW/MIA Accounting Agency_Laboratory, Joint Base Pearl Harbor-Hickam, Hawai'i 96853, USA

*Correspondence to: John E. Byrd, Defense POW/MIA Accounting Agency_Laboratory, 590 Moffet St., Bldg. 4077, Joint Base Pearl

Harbor-Hickam, Hawai'i 96853, USA

E-mail: john.e.byrd.civ@mail.mil application of existing methods to a case in which the approximate number is already known. This thoughtful analysis provides a direct assessment of performance for several approaches run against a large case. The most promising of these approaches relies heavily on pair-matching long bones, so another article addresses the accuracy of pair-matching methods directly. Here we see the impact of assemblage size on the power of visual pair-matching. Another method explored here is for predicting the pair-match of a long bone based on a set of bone measurements representing size and shape. While the predictive power exhibited is not so high as to be applied as is, it is demonstrated that there is significant information inherent in size and shape of bones that can be exploited for resolving commingling. All of this information should be exploited as far as possible.

Several articles describe ongoing large CHR problems from a variety of post-conflict scenarios. Each case has special problems to resolve and unique lessons to teach. The USS Oklahoma case includes approximately four hundred sailors killed during the December 7, 1941 attack on Pearl Harbor, Hawai'i. Due to the unique circumstances in the aftermath of the battle, the remains of all of the sailors were commingled. The scale of this CHR problem presents great challenges. However, the preservation of the remains tends to be excellent, with most bones complete and in good condition. DNA testing has proven to be very successful. This case is ideal for the development and validation of new CHR methods, since it can support such a wide variety of tests. It is no accident that the computer application Commingled Remains Analytics (CoRA) was designed using the Oklahoma case as the testbed. Like the Oklahoma case, the Battle of Tarawa case involves a single loss incident. The approximately five hundred marines and sailors missing on Tarawa died in a single battle on a small island. The similarities largely end there. The Tarawa case involves both remains buried as "unknown" in a military cemetery and remains excavated recently from the island. Thus, field provenience has a role to play along with DNA testing and other methods. In what might seem an ironic twist, DNA is readily profiled from the excavated remains, which look worse to the eye, but it can 
only be read from the "unknown" remains if next-generation sequencing (NGS) is employed. Therefore, every attempt is made to link partial remains from the field to remains from the cemetery. Remains from a former Japanese POW camp, Cabanatuan, pose greater challenges than the aforementioned as a result of their having poor preservation, mostly fragmented remains, and special DNA challenges that necessitate NGS testing as the standard test. Field provenience is somewhat useful, helping to limit the believed-to-be groups to more manageable sizes. Finally, the massive identification efforts undertaken in Bosnia and Herzegovina are well known to all in the field of forensic identification, but what is less well known are the ongoing challenges with regard to CHR cases in the extant mortuaries. The ordered reexamination of the cases by forensic anthropologists, which led to additional DNA sampling, has resulted in a substantial increase in identifications of remains that had hitherto been unidentifiable. The multidisciplinary approach described will serve as a useful guide to those contemplating DNA-led identification efforts in the future. 